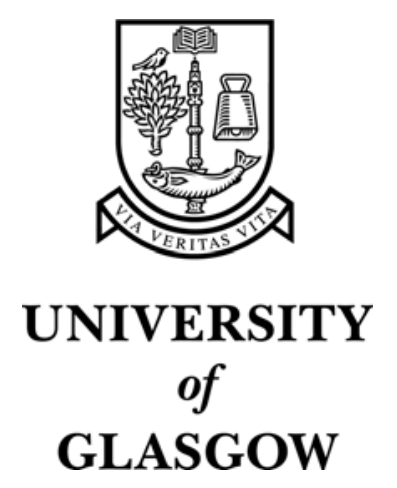

Brown, L.M. and Brewster, S.A. and Purchase, H.C. (2005) A first investigation into the effectiveness of Tactons. In, First Joint Eurohaptics Conference and Symposium on Haptic Interfaces for Virtual Environment and Teleoperator Systems, 2005., 18-20 March 2005, pages pp. 167-176, Pisa, Italy.

http://eprints.gla.ac.uk/3209/ 


\title{
A First Investigation into the Effectiveness of Tactons
}

\author{
Lorna M. Brown, Stephen A. Brewster and Helen C. Purchase \\ Glasgow Interactive Systems Group, \\ Department of Computing Science, University of Glasgow, UK \\ E-mail: \{lorna, stephen, hcp\}@dcs.gla.ac.uk \\ http://www.dcs.gla.ac.uk/ lorna
}

\begin{abstract}
This paper reports two experiments relating to the design of Tactons (or tactile icons). The first experiment investigated perception of vibro-tactile "roughness" (created using amplitude modulated sinusoids), and the results indicated that roughness could be used as a parameter for constructing Tactons. The second experiment is the first full evaluation of Tactons, and uses three values of roughness identified in the first experiment, along with three rhythms to create a set of Tactons. The results of this experiment showed that Tactons could be a successful means of communicating information in user interfaces, with an overall recognition rate of $71 \%$, and recognition rates of $93 \%$ for rhythm and $80 \%$ for roughness.
\end{abstract}

\section{Introduction}

The cutaneous sense has been under-utilised in computer interfaces, but it is known to be an effective channel through which information can be transferred, e.g. the Tadoma method as used by people who are deaf-blind shows that the cutaneous sense alone can be used to communicate complex information such as language [1]. Since vibro-tactile interfaces are becoming increasingly common in everyday devices, with mobile phones, pagers and games controllers all featuring simple vibro-tactile feedback, it is appropriate to investigate formally how best to use vibro-tactile output in human-computer interaction.

Tactons [2, 3] are structured, abstract, tactile messages which can be used to communicate information non-visually. They are the tactile counterpart of visual icons and audio earcons [4] and could be used in computer interfaces in place of, or in combination with, these. Tactons have the potential to improve interaction in a range of different areas, particularly where the visual display is overloaded, limited in size or not available, such as interfaces for blind people or in mobile and wearable devices.

The Tactons discussed in this paper are specifically vibro-tactile Tactons, but Tactons could also be created using other forms of tactile display, e.g. electro-tactile or pin arrays.

In order to create Tactons, it is necessary to identify parameters of vibration that can be used to encode information. Earcon designers use parameters such as melody, pitch and timbre (musical instrument) drawn directly from the field of music, but the tactile domain does not have an equivalent field from which to draw.

Initially, it was thought that vibro-tactile parameters such as frequency, amplitude, waveform and duration would be suitable parameters for Tacton construction [2]. However, some of these have been found to be of limited use, particularly due to the devices being used in this research (see Section 1.1). Therefore more complex parameters need to be considered. In this paper, vibro-tactile roughness and rhythm are proposed and evaluated as parameters for Tactons.

The paper starts by discussing the basic parameters of vibration, and moves on to discuss more complex parameters which could be used. Two experiments are reported; the first investigating perception of vibrotactile "roughness", in order to assess its potential as a Tacton parameter, while the second is the first investigation into Tacton design and evaluates Tactons which use roughness and rhythm as parameters.

Throughout the paper comparisons are drawn between Tactons and earcons, since the fields are very similar, and it may therefore be beneficial to use earcon design to inform the initial design of Tactons.

\subsection{Vibro-tactile devices used in this research}

Two vibro-tactile devices were used in this research: the Audiological Engineering Corporation (AEC) TACTAID VBW32 transducer (www.tactaid.com) and the Engineering Acoustics Inc 
(EAI) C2 Tactor (www.eaiinfo.com). Each TACTAID transducer costs around US\$80 while the C2s Tactors are more expensive, costing around US\$230.

The TACTAID VBW32 (Figure 1) is the transducer used in AEC's tactile hearing aids. It is an inertial transducer, which consists of a rigid case, inside which a mass is suspended on a spring (Figure 2). Both the mass and the case vibrate when an alternating electromagnetic force is generated [5], so the user feels the vibrations through the case itself.

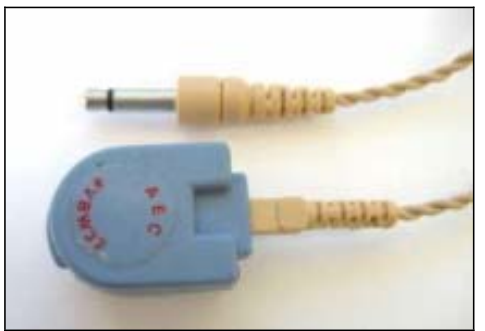

Figure 1: AEC TACTAID VBW32 with $3.5 \mathrm{~m}$ jack.

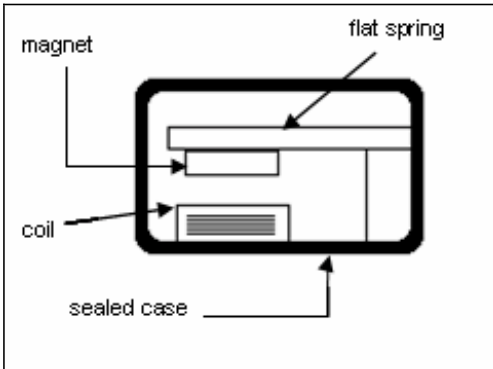

Figure 2: Construction of an inertial transducer similar to the TACTAID (adapted from [5]).

The C2 Tactor (Figure 3) is a voice coil transducer. In this type of device the contactor (contact point with the skin) is located outside the case, therefore the user only feels the vibration through the contactor. A typical voice coil transducer design is shown in Figure 4. This uses a similar construction to audio speakers. In such a device, the coil is located in a magnetic field, and when a current passes through the coil, the coil is pushed along its axis, causing the contactor to vibrate [5].

Both of these devices are resonant at $250 \mathrm{~Hz}$ and have very reduced output at other frequencies. The results reported in this paper are specific to these devices and may not transfer to devices with a wider bandwidth. These devices were chosen since they are commercially available, small, and portable, and could therefore be used in mobile or wearable devices.

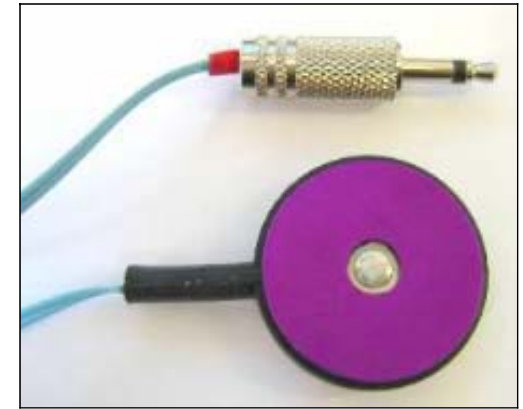

Figure 3: EAI C2 Tactor with 3.5mm jack.

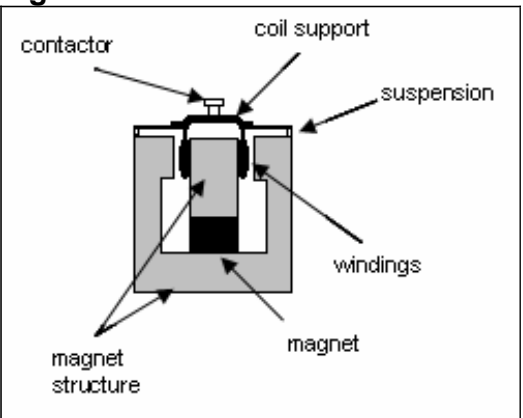

Figure 4: Typical construction of a voice-coil (shaker) transducer (adapted from [5]).

\section{Vibro-Tactile Parameters}

Before Tactons can be created, appropriate vibrotactile parameters in which information can be encoded must be identified. This section discusses the basic parameters of vibration, and then suggests some more complex parameters which may be of greater use in Tacton design.

\subsection{Basic vibro-tactile parameters}

The most obvious parameters to use in Tacton construction are the basic parameters of vibration such as frequency, amplitude, waveform and duration. These parameters and their limitations for use in Tactons, are discussed in this section.

2.1.1 Frequency. While humans can hear sounds in the range $20-20,000 \mathrm{~Hz}$, the practical frequency range of the skin is much smaller, ranging from $10 \mathrm{~Hz}$ to $400 \mathrm{~Hz}$ [5]. The usable frequency range is further reduced by the limited bandwidth of the devices used (see Section 2.1), making frequency modulation alone unusable in Tacton design with these devices.

2.1.2 Amplitude. In earcons, amplitude is not used as a parameter because users find loud sounds annoying 
and report annoyance when the volume level is out of their control [6]. Using amplitude as a parameter in Tactons could be equally problematic as reducing the amplitude could degrade perception of other parameters, or render the signal undetectable, while increasing it too far could cause pain [7]. Therefore, it is best to leave amplitude under the control of the user.

2.1.3 Waveform. Waveform (or timbre) is one of the most important parameters of earcons, but may not be so useful in Tactons due to the fact that subtle differences in waveform cannot be perceived on the skin. In addition, few tactile waveforms could be used without some training unlike in audio where musical instruments provide a wide range of easily recognisable timbres.

A further constraint on the use of waveforms is the limited bandwidth of the devices used in this research, which mean that differences in waveforms are lost. Figure 5 shows the output from the TACTAID when a sine wave is played through it, while Figure 6 shows the output when a square wave is played - all but three of the frequency components of the square wave are lost due to the limited bandwidth of the TACTAID.

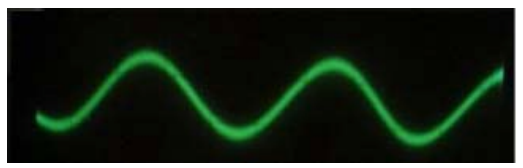

Figure 5: Output of TACTAID when a sine wave was played through it.

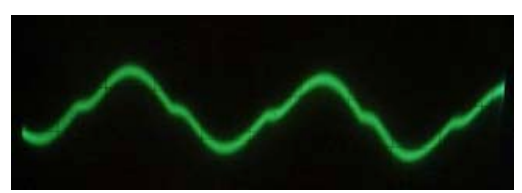

Figure 6: Output of TACTAID when a square wave was played through it.

2.1.4 Duration. Information could be encoded by manipulating the duration of pulses. While duration alone could be used as a parameter, combining pulses of different durations to form rhythms (as discussed in Section 2.2.2) would offer more flexibility.

\subsection{Possibilities of more complex parameters}

Due to the limitations of the basic parameters of vibration it is necessary to consider more complex parameters in order to encode information in Tactons. Some possible parameters are discussed below.
2.2.1 Rhythm. Rhythm is an extremely important parameter in earcon design and could be equally, if not more, important in Tactons. Rhythms can be created by grouping together pulses of different durations. Summers [8] used temporal patterns (rhythms) along with frequency and amplitude to encode speech information in vibrations, and found that participants mainly used the information obtained from the temporal patterns, rather than from the frequency/amplitude modulation. This suggests that rhythm could be an important parameter in Tacton design. This is discussed in more detail in Section 4.

2.2.2 Complex waveforms. Section 2.1.3 discussed the problems of recognition of simple waveforms such as square waves and sine waves. Therefore it was necessary to consider more complex waveforms. Experimentation with the vibro-tactile transducers described in Section 1.1. indicated that perceptually different waveforms could be created using sinusoidal amplitude modulation. Amplitude modulated signals are created by multiplying a sine wave of a given frequency by a sine wave of another frequency, thus modulating the amplitude of the base signal by the second frequency. Figure 7 shows a $250 \mathrm{~Hz}$ sinusoid modulated by a $30 \mathrm{~Hz}$ sinusoid.

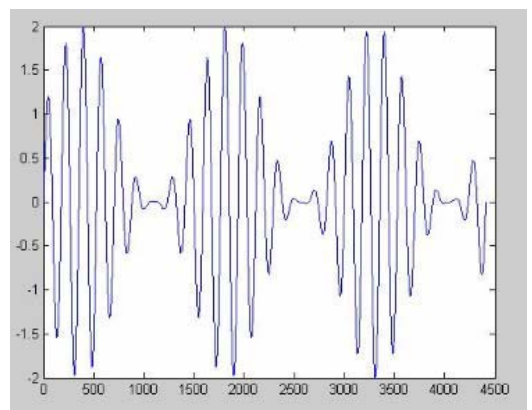

Figure 7: $250 \mathrm{~Hz}$ sinusoid modulated by a $30 \mathrm{~Hz}$ sinusoid.

2.2.3 Spatial Location. The spatial location of transducers on the body has been used successfully by many researchers, such as van Veen and van Erp [9] for communicating information. This is not used as a parameter in either of the experiments presented in this paper, but will be considered in future work.

\subsection{Recognition of Abstract Parameters}

In addition to finding parameters that people can distinguish, it is also important to find parameters that people are able to label and, therefore, remember. In audio this is made simpler by the existence of music, 
as labels such as instrument (e.g. trumpet/piano/violin), pitch (high/low), and melody are familiar to most people and can be used in earcons with little explanation. Brewster [10] showed that using musical timbres in earcons was more effective than using simple tones, e.g. sine waves, square waves, and it is likely that this was at least partly due to the difficulty in labeling such abstract tones, compared to the ease of recognising a musical instrument, e.g. trumpet, piano.

Many vibro-tactile parameters are abstract and unfamiliar, so it is very important to find a way that people can label the values of these parameters. One such parameter is amplitude modulation. To a nonscientist this term may be meaningless, and even people familiar with the term would be unlikely to have any idea what such a waveform would feel like.

Previous research has indicated a relationship between amplitude modulation and roughness, suggesting that roughness may be an appropriate term to label amplitude modulated vibro-tactile signals. When Weisenberger [11] presented subjects with pairs of stimuli, one an un-modulated sinusoid and the other an amplitude modulated version of the same sinusoid, subjects reported the task as being a roughness detection task, where modulated sinusoids felt "rougher" than the un-modulated sinusoid. This is supported by research in audio, in which the relationship between amplitude modulation and roughness is well established $[12,13]$.

\section{Experiment 1: Roughness Perception}

Based on the indications that roughness was an appropriate term to describe amplitude modulated signals, an experiment was run to determine whether people could differentiate between five different amplitude modulated signals in terms of roughness. The aim was to identify three or more values of roughness that could be used in Tacton design, so it was necessary to find out whether there were three values which people consistently labeled as rougher or smoother than each other, i.e. a stimulus that could be labeled "smooth", one that could be labeled "rough" and another that could be labeled "very rough".

While it would have been possible to simply choose three values and assign them to roughness labels, studies in force-feedback suggest that perception of roughness differs greatly between individuals [14], and it was not known whether these individual differences might also occur in the vibro-tactile domain. Therefore, one aim of this experiment was to investigate whether peoples' perception of vibro-tactile roughness was consistent.

\subsection{Stimuli}

Five stimuli were used in this experiment; all modulations of a $250 \mathrm{~Hz}$ sine wave. $250 \mathrm{~Hz}$ was chosen as the base frequency for all stimuli as it is the peak response frequency of both devices, and also the frequency at which the skin is most sensitive. The stimuli used were sine (no modulation), $\bmod 20$ (the same sine wave modulated by $20 \mathrm{~Hz}), \bmod 30$ (modulated by $30 \mathrm{~Hz}$ ), mod40 (modulated by $40 \mathrm{~Hz}$ ), and $\bmod 50$ (modulated by $50 \mathrm{~Hz}$ ).

$20 \mathrm{~Hz}$ was chosen as the lowest modulation frequency based on results from the audio domain. Terhardt [12] reported that below $20 \mathrm{~Hz}$ people recognise the individual fluctuations within a signal, whereas above that point the individual fluctuations are no longer perceived as separate events, and the signal sounds "rough" or "harsh". Informal pilot testing confirmed that $20 \mathrm{~Hz}$ was considered to feel "rough", rather than like individual pulses. These pilots also indicated that the vibration started to feel smooth above $50 \mathrm{~Hz}$ so this was chosen as the highest modulation frequency.

\subsection{Hypotheses}

1. Participants will be able to discriminate between the stimuli based on roughness.

2. Sine wave with no modulation will be perceived as smoother (less rough) than all other stimuli.

3. Apart from the un-modulated sine wave, the lower the modulation frequency, the "rougher" the stimuli will be perceived.

\subsection{Vibro-tactile devices}

The experiment was run twice, once on each of the two vibro-tactile transducers described in Section 1.1. This allowed a comparison between an inexpensive device (TACTAID) and a more sophisticated device (C2).

The transducer was attached to the index finger of the participant's non-dominant hand using double sided sticky tape, and secured using surgical tape (Figure 8). The finger-tip was selected as the location for stimulation since it is very sensitive to vibrations and therefore provides a best-case-scenario. Future work will look at how these stimuli transfer to different body locations, such as the wrists or the back. These sites may be more practical for real world applications as they would allow users to keep their hands free for other tasks. 
Participants were asked to keep their hand palmside up as much as possible in order to keep the intensity of the vibrations consistent. They could rest their arm on the arm rest attached to the desk, or on their leg.

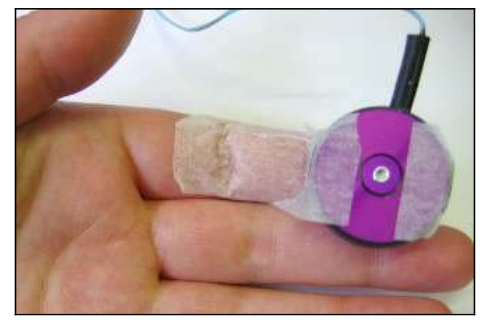

Figure 8: C2 Tactor attached to finger.

\subsection{Design}

3.4.1 Participants. 18 participants took part in this experiment, with nine different participants used for each device. All participants were students or staff from the University of Glasgow ( 5 female, 13 male, all right handed), and were paid $£ 5$ for their participation.

3.4.2 Experimental Procedure. The experiment consisted of 50 tasks, and used a forced choice design. In each task participants were asked to compare two stimuli and indicate which stimulus felt "rougher". Participants could choose to feel each stimulus up to four times. Once they had made their decision, they indicated their response by clicking on the corresponding radio button (on the dialogue shown in Figure 9). Every possible pairing of stimuli was presented four times, with the order of presentation randomised.

Before starting the experiment participants were trained to use the interface by performing four tasks from the experiment. They received no feedback on their performance during training, and did not receive any training on what was meant by "roughness", as the aim was to find out how people intuitively perceive roughness, rather than to train them to perceive certain stimuli as rougher than others.

\subsection{Results}

During the experiment, data were collected on participants' responses to each pair of stimuli. These data were analysed by considering every pair in turn and looking at how many times each stimulus was considered to be rougher than each other stimulus. This analysis was performed using pair-wise Wilcoxon tests.

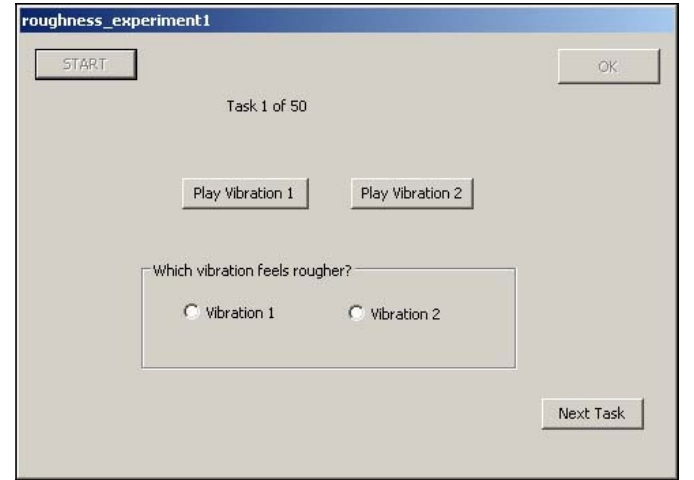

Figure 9: The roughness experiment dialogue.

3.5.1. Results: AEC TACTAID VBW32. The results for the TACTAID showed a significant difference between the un-modulated sine wave and all other stimuli $(T=0(n=9) ; p<0.01)$, indicating that all other stimuli were perceived to be rougher than the unmodulated sine wave. In addition there was a significant difference between $\bmod 40$ and $\bmod 50$ $(\mathrm{T}=2.5(\mathrm{n}=8) ; \mathrm{p}<0.05)$ with $\bmod 40$ considered to be rougher than mod50. No other significant differences were shown. After the experiment, four participants reported confusion with the concept of roughness in terms of how it related to these vibrations. They referred to the "speed", "intensity" and "frequency of the vibrations, rather than the roughness.

3.5.2 Results: EAI C2 Tactor. As with the TACTAID, the results for the C2 Tactor showed a significant difference between the un-modulated sine wave and all other stimuli. In addition, significant differences were found for all other pairs, except $\bmod 20$ and $\bmod 30$. The results can be expressed as followed, with " $>$ " indicating that the stimulus on the left was perceived to be rougher than the stimuli on the right.

- $\bmod 20>\bmod 40(\mathrm{~T}=1.5(\mathrm{n}=8), \mathrm{p}<0.05)$

- $\bmod 20>\bmod 50(\mathrm{~T}=1(\mathrm{n}=9) ; \mathrm{p}=0.01)$

- $\bmod 20>\operatorname{sine}(\mathrm{T}=1(\mathrm{n}=8) ; \mathrm{p}=0.01)$

- $\bmod 30>\bmod 40, \bmod 50, \operatorname{sine}(\mathrm{T}=0(\mathrm{n}=9) \mathrm{p}<0.01)$

- $\bmod 40>\bmod 50(\mathrm{~T}=0(\mathrm{n}=8) ; \mathrm{p}=0.01$

- $\bmod 40>\operatorname{sine}(\mathrm{T}=0(\mathrm{n}=9) ; \mathrm{p}<0.01)$

- $\bmod 50>\sin e(T=0(n=9) ; p<0.01)$

These results indicate that participants felt that roughness increased as modulation frequency decreased, with the exception of the un-modulated sine wave, which felt less rough than all other stimuli. These results are much more consistent than those for 
the TACTAID. In addition, no participants mentioned a problem with the use of the term "roughness" in relation to the stimuli, unlike when using the TACTAID. However, this is not quantifiable, and may simply be due to the nature of the participants and their willingness to discuss their experience.

\subsection{Discussion}

The results for the TACTAID indicate that, using this device, there are three stimuli which can be differentiated in terms of roughness, namely sine, $\bmod 40$ and $\bmod 50$.

If using the $\mathrm{C} 2$ Tactor, four stimuli can be differentiated in terms of roughness. There are two options for these four stimuli: either sine, $\bmod 20$, $\bmod 40$ and $\bmod 50$, or $\operatorname{sine}, \bmod 30, \bmod 40$ and $\bmod 50 . \bmod 20$ and $\bmod 30$ were not distinguishable in terms of roughness, therefore should not both be used together.

Hypothesis 1 can be accepted for the $\mathrm{C} 2$ Tactor as all but one pair of stimuli were found to be distinguishable. It can also be partially accepted for the TACTAID as several pairs were distinguishable.

Hypothesis 2 can be accepted for both devices, as the un-modulated sine wave was always perceived to be smoother (or less rough) than all other stimuli. Hypothesis 3 can be accepted for the $\mathrm{C} 2$ Tactor, in that for those stimuli which were distinguishable (all but one pair), roughness increased as modulation frequency decreased. With so few pairs of stimuli distinguishable on the TACTAID (only one pair other than those paired with sine) there are not enough data points to accept or reject this hypothesis for the TACTAID.

3.6.1 Difference between the devices. The results of this experiment are very different for each device with all but one pair showing a significant difference on the C2 Tactor, and only 5 of the 10 pairs showing significance on the TACTAID (and only one of these which did not include the un-modulated sinewave). The C2 Tactor also showed a consistent ordering in terms of roughness.

It is possible that this difference is due to the limitations of the study. With only nine participants in each condition, and a between-groups design, this result could be due to individual differences rather than a difference in the devices. However, there are several other explanations.

One possible explanation is that the difference is due to the nature of stimulation of the two devices, i.e. while the TACTAID only stimulates the rapidly adapting receptors (as the whole case vibrates), the $\mathrm{C} 2$ also indents the skin when it vibrates, therefore it may also stimulate the slowly adapting receptors.

A simpler explanation may be that the difference is due to the different response times of the two devices. The $\mathrm{C} 2$ responds much more quickly to changes than the TACTAID therefore the TACTAID may have attenuated the intensity and the modulation depth, making the roughnesses less distinguishable.

Since the C2 Tactor was found to be more successful in this experiment, future Tacton experiments will use this device.

\subsubsection{Implications for Tacton Design.}

The results of this experiment only indicate which stimuli can be distinguished from one another; they do not indicate whether each stimulus could be uniquely identified (which will be necessary for designing Tactons). Therefore the next experiment (Section 4) aimed to look at whether several of these stimuli could be uniquely identified when used, along with another parameter (namely rhythm) in the context of Tactons.

\section{Experiment 2: Evaluation of Tactons}

The results from Experiment 1 showed that the participants were able to distinguish between different amplitude modulated signals in terms of roughness, so the next step was to implement several of these "roughnesses" as parameters in Tactons.

Although Tactons could be created from just a single parameter, more information can be encoded when several parameters are combined. In this experiment it was decided that two parameters would be used, and rhythm was chosen as a second parameter since it has been successful in earcons, and has been shown to be useful in other tactile interfaces [8].

Various types of Tactons have been proposed, namely Compound Tactons, Hierarchical Tactons, and Transformational Tactons [2]. For this experiment, Transformational Tactons (the tactile equivalent of Transformational Earcons [4]) were created to represent alerts which might occur when a message or a call arrives on a mobile phone. Transformational Tactons represent several attributes at once, with each encoded in a different tactile parameter. Two pieces of information were encoded in each call/message Tacton: the type of call or message (voice call, text message, or multimedia message) was encoded in the rhythm, while the priority (low, medium or high) of the call or message was encoded in the roughness. Using this mapping, the same rhythm would represent a high priority voice call and a low priority voice call but they 
would each be presented using a different roughness, whereas a high priority voice call and a high priority text message would share the same roughness, but have different rhythms.

\subsection{Aim and Hypotheses}

The aim of this experiment was to investigate absolute identification of Tactons. Data were recorded on the recognition of complete Tactons (correct identification of both call/message type and priority), in addition to the recognition of the individual attributes. The hypotheses were:

1. Participants will be able to identify different rhythms, representing type of call/message.

2. Participants will be able to identify different roughnesses, representing call/message priority.

3. Participants will be able to identify complete Tactons

This experiment is the first evaluation of Tactons, and the results will therefore provide a base point for Tacton recognition rates in future, and provide some indication of whether Tactons could be successful.

\subsection{Vibro-tactile device}

The results of Experiment 1 indicated that the $\mathrm{C} 2$ Tactor would be more suitable for creating Tactons than the TACTAID, as people were able to distinguish between more pairs of stimuli in terms of roughness. Therefore the $\mathrm{C} 2$ Tactor was used in this experiment. The device was attached to each participant's index finger in the same way as in Experiment 1.

\subsection{Stimuli}

Each parameter in this experiment had three values (e.g. low/medium/high), and this section outlines how these values were selected. This number of values was chosen because it has been shown in earcon design that people are able to remember and identify three different values of each parameter [15].

4.3.1 Roughness. Three distinct roughnesses were required for this experiment, to represent low (smooth), medium (rough) and high (very rough) priority calls/messages. Based on the results of Experiment 1, three amplitude-modulated signals were chosen, and mapped to a roughness label. These were sine (smooth), mod50 (rough) and mod30 (very rough). The reasons for this choice of stimuli are explained below.
Experiment 1 identified that four different values of roughness were distinguishable when using the $\mathrm{C} 2$ Tactor. Since only three values were required for this experiment it was necessary to pick the three stimuli which were most likely to be uniquely identifiable.

The un-modulated sine wave was discriminable from all other stimuli so was selected. For the other stimuli it seemed logical that using stimuli that were further apart in frequency would increase the chance of absolute recognition, therefore either $\bmod 20$ and $\bmod 40$, or $\bmod 30$ and $\bmod 50$ would be suitable choices. Since the difference between $\bmod 20$ and mod40 had a higher probability $(\mathrm{p}<0.05)$ of being due to chance than the difference between $\bmod 30$ and $\bmod 50(\mathrm{P}<0.01)$, this pair was rejected and $\bmod 30$ and mod50 were used.

Another reason for choosing $\bmod 30$ rather than $\bmod 20$ is due to the speed of the fluctuations of the amplitude modulation. The lower the modulation frequency, the slower the fluctuations will be, and the longer it will take for people to perceive the roughness. Since Tactons should be as short as possible in order to communicate information quickly, it is important to choose higher modulation frequencies, so that shorter pulses can be used.

4.3.2. Rhythm. Three different rhythms were created to represent the three types of message: voice call, text message and multimedia message. While the roughness stimuli were based on the results of the previous experiment, there was no previous experiment evaluating suitable rhythms for Tactons, so the rhythms were designed based on Brewster's guidelines for rhythms in earcons [10]. In order to make each rhythm feel as different as possible a different number of notes (pulses) was used in each rhythm. These rhythms are presented in Figures 10-12 using standard musical notation for rhythm, on a single line since no pitch information is required.

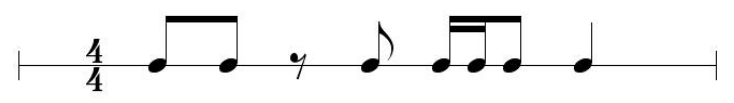

Figure 10: Voice Call Rhythm.

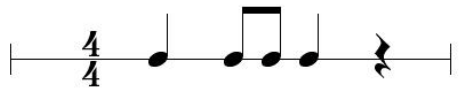

Figure 11: Text Message Rhythm.

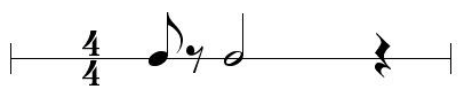

Figure 12: Multimedia Message Rhythm. 
In addition to following Brewster's guidelines, these rhythms also follow advice given by van Erp and Spapé [16] who identified tempo (speed) as an important parameter in the identification of tactile melodies. Although all three rhythms are created using the same tempo, they feel faster or slower due to the use of many short notes (e.g. voice call), or few long notes (multimedia message).

\subsection{Design}

4.4.1 Participants. 17 participants (staff and students of the University of Glasgow, 4 female, 13 male, all right handed) took part in this experiment and were paid $£ 5$ for their participation.

4.4.2 Experimental Procedure. Before starting the experiment participants were trained to recognise the different Tactons. The way in which the data were encoded in the Tactons was explained, and then the participants were given 10 minutes to explore a $\mathrm{Web}$ page on which all of the Tactons used in the experiment were provided. After the 10 minutes they took part in 18 tasks from the experiment itself as training in how to use the interface. This extended training aimed to eliminate any learning effect during the experiment itself. Pilot participants (who did not receive this training) had reported that they became more confident in their responses after around 18 tasks; before this point they still felt they were learning.

The experiment itself consisted of 54 tasks, consisting of six presentations of every Tacton. In each task participants were presented with one Tacton, which was repeated four times with a one second pause between repetitions. While the Tacton was being presented, participants had to identify both of the attributes (the type and priority of the call/message) encoded in the Tacton, and indicate their responses by clicking on the corresponding radio buttons (Figure 13). They could respond as quickly as they wanted; they did not need to wait until the Tacton has been repeated all four times.

\subsection{Results}

During the experiment data were collected on the number of correct responses to the type and priority of each Tacton. Percentage correct scores were calculated for each individual attribute (type and priority) and for the complete Tactons. These three sets of data were analysed individually using analyses of variance (ANOVAs).

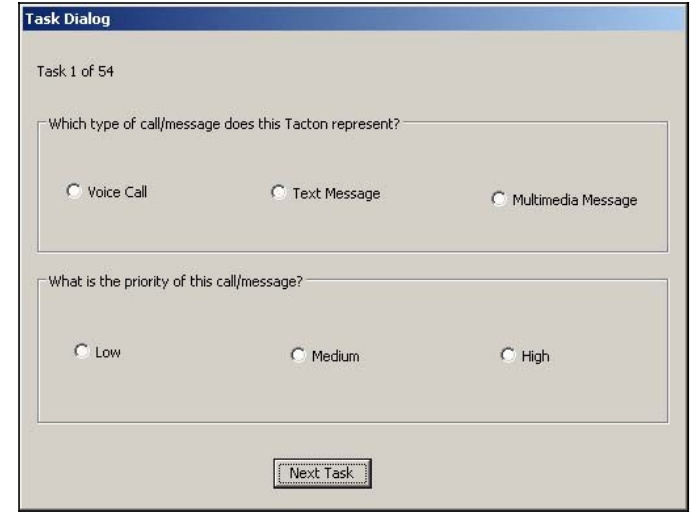

Figure 13: The Tacton experiment dialogue.

One participant was eliminated from the evaluation as he confused the mapping between rhythm and call type, and consistently answered using the wrong mapping. Therefore the results are only reported for the remaining 16 participants.

The results for overall Tacton recognition (Figure 14) showed an average recognition rate of $71 \%$, with high priority multimedia messages having the highest recognition rate (86\%), and medium and high priority voice calls having the lowest (both 60\%). There were no significant differences in recognition rates between the different Tactons $(\mathrm{F}(8,135)=1.41, \mathrm{p}=0.199)$.

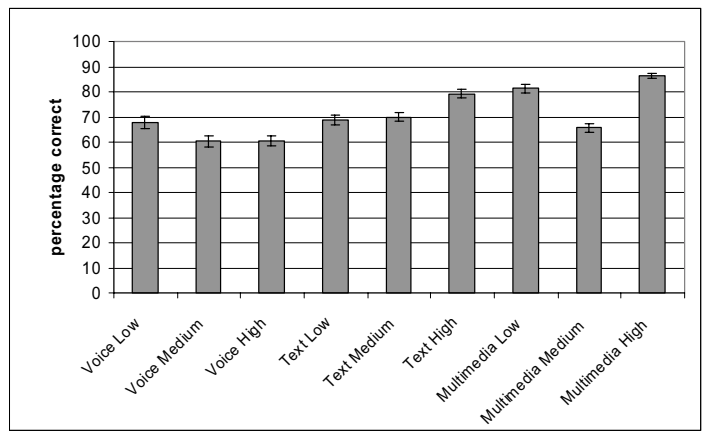

Figure 14: Percentage Correct: overall Tacton recognition (with standard deviations).

Call/message types (represented by rhythm) were correctly recognised on average $93 \%$ of the time (Figure 15), with multimedia messages being best recognized $(99 \%)$ and voice calls having the lowest recognition rate $(86 \%)$. There were no significant differences in the recognition rates $(\mathrm{F}(2,45)=1.83$, $\mathrm{p}=0.172$ ). Informal discussion with participants after the experiment revealed that several participants felt that they had difficulty distinguishing between the voice call rhythm and the rhythm representing a text 
message. Although there is no significant difference, the results do show slightly lower recognition of both of these rhythms than multimedia messages.

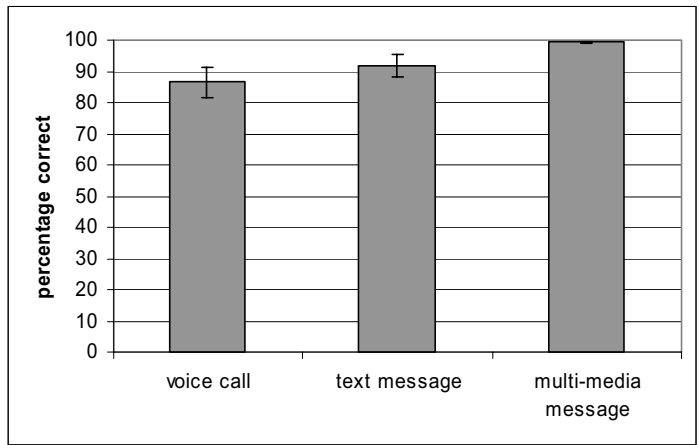

Figure 15: Percentage Correct: Type of Call/Message (with standard deviations).

The results for recognition of priority of call/message (represented by roughness) showed an average recognition rate of $80 \%$ (Figure 16), with low priority having the highest recognition rate $(82 \%)$ and medium priority the lowest $(76 \%)$. There were no significant differences in the recognition rates (F $(2,45)=0.34, p=0.711)$. Informal feedback indicated that many participants felt they often got confused between the medium and high priorities, although the results do not reflect this.

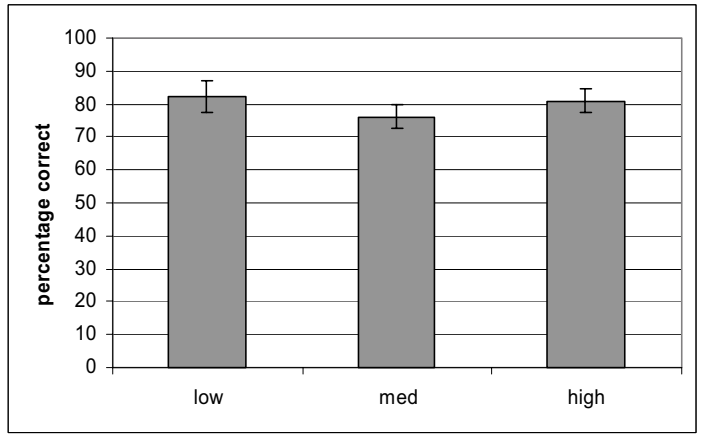

Figure 16: Percentage Correct: Priority of Call/Message (with standard deviations).

\subsection{Discussion}

The results of this experiment seem very promising for Tactons. The overall recognition rate of $71 \%$ compares favourably to results for earcon recognition, where McGookin's evaluation of single Transformational earcons [15] showed overall recognition of around $70 \%$ (although he used three parameters rather than just two so a direct comparison cannot be made). Looking at the individual parameters, McGookin found a recognition rate of over $90 \%$ for melody, which matches the recognition rate for rhythm in Tactons (93\%), indicating that rhythm will be a very useful parameter in Tactons. The roughness parameter in Tactons achieved $80 \%$ recognition, which is lower than McGookin's result for timbre (over 90\%), but higher than the result for his third parameter, register $(75 \%)$. This indicates that roughness will be a usable parameter in Tactons, although perhaps not as good as rhythm.

\section{Conclusions and Future Work}

This paper described two experiments; the first investigating perception of vibro-tactile roughness with a view to identifying values of roughness which could be used in Tactons, and the second evaluating a set of Tactons created using roughness and rhythm as parameters.

The first experiment showed the participants were able to differentiate between amplitude modulated sinusoids in terms of roughness. Three roughnesses were selected for use in the second experiment: an unmodulated $250 \mathrm{~Hz}$ sine wave (smooth), and the same sine wave modulated by $50 \mathrm{~Hz}$ (rough) and $30 \mathrm{~Hz}$ (very rough).

These roughnesses were used, along with three rhythms, to create a set of Tactons, which were evaluated in the second experiment. This was the first full evaluation of Tactons, and provides a basis from which to design future Tactons, and a result to which future Tacton experiment results can be compared.

The second experiment showed $71 \%$ unique identification of Tactons, which is comparable to earcon results $(70 \%)$. The rhythms were correctly identified over $90 \%$ of the time, indicating that rhythm will be a very successful parameter. With one rhythm achieving $99 \%$ recognition, it may even be possible to increase the number of rhythms used. Future experiments could look at other ways of creating rhythms which may make them even more distinguishable, such as using distinctive musical styles (e.g. Latin, Swing, March) for each rhythm.

The results also indicate that roughness could be a usable parameter for Tactons, as it achieved a recognition rate of $80 \%$, which is comparable to that achieved by register (pitch) in earcons (around 75\%). One problem reported by participants regarding roughness identification was that identification was more difficult when rhythms containing short notes (e.g. voice call rhythm) were used. This may be due to 
the fact that there is less time to identify the roughness when the pulses are shorter. Future work could look at identifying the minimum duration at which these roughnesses are identifiable, and rhythms could then be designed around these results.

In order to improve all the results, it might be worth considering providing feedback on performance during training. As reported in Section 4.3, one subject confused the mapping from rhythm to type, but this confusion might have been resolved if the subject had received feedback about whether his answers were correct. In addition, several subjects reported that they found it difficult to judge between medium and high priorities. Providing feedback during training could help to give users confidence in their responses.

All these results refer to Tactons containing two parameters. Earcons have been created using three parameters, and ideally Tactons should be able to replicate this. Future work should investigate the effect on the current parameters of adding a third parameter.

Overall, the results indicate that Tactons could be an effective means of communicating information in user interfaces, and suggest that further work in this area would be worthwhile.

\section{Acknowledgements}

This work is funded by EPSRC studentship GR/PO1175/01 and EPSRC grant GR/S53244. Thanks to Roger Cholewiak for his input to this work, in particular for the information on the TACTAID and C2 Tactors.

\section{References}

[1] Tan, H.Z. and Pentland, A., "Tactual displays for sensory substitution and wearable computers." Fundamentals of wearable computers and augmented reality, W. Barfield and T. Caudell, eds., Lawrence Erlbaum Associates: Mahwah, New Jersey, 2001. pp 579-598.

[2] Brewster, S. and Brown, L.M. "Tactons: Structured Tactile Messages for Non-Visual Information Display", in Proceedings of AUIC 2004, Dunedin, New Zealand: Australian Computer Society, pp 15 - 23.

[3] Brewster, S.A. and Brown, L.M. "Non-Visual Information Display Using Tactons", in Extended Abstracts of ACM CHI 2004, Vienna, Austria: ACM Press, pp 787-788.

[4] Blattner, M.M., Sumikawa, D.A., and Greenberg, R.M., "Earcons and Icons: Their Structure and Common Design Principles", Human Computer Interaction 4(1), 1989, pp. 11-44.
[5] Cholewiak, R.W. and Wollowitz, M., "The design of vibrotactile transducers", Tactile Aids for the Hearing Impaired, I. Summers, ed, Whurr Publishers Ltd: London, 1992. pp 57-82.

[6] Brewster, S.A. and Crease, M.G., "Correcting Menu Usability Problems With Sound", Behaviour and Information Technology 18(3), 1999, pp. 165-177.

[7] Craig, J.C. and Sherrick, C.E., "Dynamic Tactile Displays", Tactual Perception: A Sourcebook, W. Schiff and E. Foulke, eds., Cambridge University Press, 1982. pp 209-233.

[8] Summers, I.R. "Single Channel Information Transfer Through The Skin: Limitations and Possibilities", in Proceedings of ISAC 2000, University of Exeter, UK.

[9] van Veen, H.A.H.C. and van Erp, J.B.F., "Tactile Information Presentation in the Cockpit." Haptic Human-Computer Interaction, S. Brewster and R. Murray-Smith, eds., LNCS 2058, Springer: Berlin, 2000. pp 174-181.

[10] Brewster, S.A., "Providing a Structured Method for Integrating Non-Speech Audio into Human-Computer Interfaces", PhD Thesis, University of York, UK, 1994

[11] Weisenberger, J.M., "Sensitivity to amplitudemodulated vibrotactile signals", Journal of the Acoustical Society of America 80(6), 1986, pp. 17071715.

[12] Terhardt, E., "On the perception of periodic sound fluctuations (roughness)", Acustica 30, 1974, pp. 201213.

[13] Fastl, H., "Roughness and temporal masking patterns of sinusiodally amplitude modulated broadband noise", Psychophysics and Physiology of Hearing, E.F. Evans and J.P. Wilson, eds., Academic: London, 1977. pp 403414.

[14] Penn, P., et al., "The Haptic Perception of Texture in Virtual Environments: An Investigation with Two Devices", Haptic Human Computer Interaction, S Brewster and R. Murray-Smith, eds., LNCS 2058, Springer: Berlin, 2000. pp 25-30.

[15] McGookin, D.K. and Brewster, S.A., "Understanding Concurrent Earcons: Applying Auditory Scene Analysis Principles to Concurrent Earcon Recognition", ACM Transactions on Applied Perception 1(2), 2004, pp. 130155.

[16] van Erp, J.B.F. and Spapé, M.M.A. "Distilling the Underlying Dimensions of Tactile Melodies", in Proceedings of Eurohaptics 2003, Dublin, Ireland, pp 111-120. 